\title{
The value of hysteroscopic-based decision-making in uterine abnormalities during tamoxifen intake in breast cancer patients - preliminary report
}

\author{
Iwona Gabriel, Anita Olejek \\ Department of Gynecology, Obstetrics and Oncological Gynecology, Medical University of Silesia, Katowice, Poland
}

Videosurgery Miniinv 2014; 9 (2): 258-261

DOI: $10.5114 /$ wiitm.2013.40106

\begin{abstract}
Women using hormonal therapy for breast cancer are often encountered in clinical practice of breast surgeons, oncologists and gynecologists. Some of them during the course of therapy develop abnormal uterine bleeding or have ultrasound abnormalities detected. In Poland, most of them are still diagnosed using blind curettage, which does not bring a definitive diagnosis or requires repeating the diagnostic procedure. This produces unnecessary fear of malignancy in patients and increases economic costs (double curettage, unnecessary hysterectomy and its social consequences). Therefore, we studied the usefulness of hysteroscopy with targeted biopsies for further management of women treated with tamoxifen. Our goal is to provide evidence that women before entering, during and after tamoxifen treatment have hysteroscopy for endometrial assessment.
\end{abstract}

Key words: tamoxifen, hysteroscopy, breast cancer, endometrium, assessment.

\section{Introduction}

The long-term effect of tamoxifen treatment in breast cancer patients is widely known. These patients are carefully followed during tamoxifen treatment by their oncologists, but they rarely have their baseline endometrial assessment performed by their gynecologist. A recent Spanish study proved the value of hysteroscopic assessment prior to treatment even in asymptomatic postmenopausal women with ER-positive receptors [1]. These findings are crucially important in the case of obese, older women whose risk of endometrial pathology is primarily greater than the population risk.

In Poland, there is no follow-up schema for qualification and follow-up of patients with breast cancer treated with tamoxifen. The main tool to assess the potential risk of uterine pathology is the clini- cal examination and transvaginal ultrasound (TVS). Recent comparative studies of hysteroscopy vs. TVS showed $63.6 \%$ specificity, $81.8 \%$ sensitivity, $72.9 \%$ positive predictive value and $73.7 \%$ negative predictive value of TVS, whereas corresponding values for diagnostic hysteroscopy were 100\%, 98.1\%, 100\% and $97.8 \%$ [2]. The important finding was that 38 patients (39.2\%) who were tested negative in TVS appeared to have uterine pathology (10 patients had polyps, 3 had hyperplasia and 25 had atrophy). On the other hand, 59 patients were tested positive in TVS. Forty-three patients had polyps, 4 had hyperplasia and 25 atrophy [3]. Both of these studies selected asymptomatic patients.

Therefore we aimed to start a procedure of hysteroscopic assessment of endometrium in collaboration with an oncology unit for women treated with tamoxifen, whether they were symptomatic 
(AUB) or asymptomatic (suspicion of uterine pathology on TVS).

\section{Case report}

Premenopausal and postmenopausal women treated with tamoxifen are recruited to the study. They are divided according to a previous study [4] into 4 subgroups (premenopausal - less than 3 years of tamoxifen intake - IA, more than 3 years - IB; postmenopausal - less than 3 years of tamoxifen intake - IIA, more than 3 years - IIB). After the written informed consent is signed, hysteroscopy under intravenous sedation using a diagnostic hysteroscope is performed (Storz, Germany) with direct sampling of the endometrial stripe. It is provided as 1-day hospital stay.

Nine women aged 51-65 have been recruited so far. All of the women received tamoxifen longer than 3 years (4 of them for more than 48 months). Seven of them were referred to the department due to suspected hyperplasia in TVS, 1 had pyometra and 1 AUB.

Four of 7 women with suspected hyperplasia had atrophic endometrium, 2 of 7 had simple hyperplasia and 1 of 7 had endometrial polyp (non-malignant). One woman who had suspicion of pyometra had atrophic endometrium with non-malignant fluid collection due to cervical stenosis. The cavitary fluid was sent for bacterial analysis revealing enterococci. One woman with abnormal uterine bleeding had an endometrial polyp that was removed and showed no malignancy. However, after a change of hormone therapy for anastrozole, she began to have abnormal

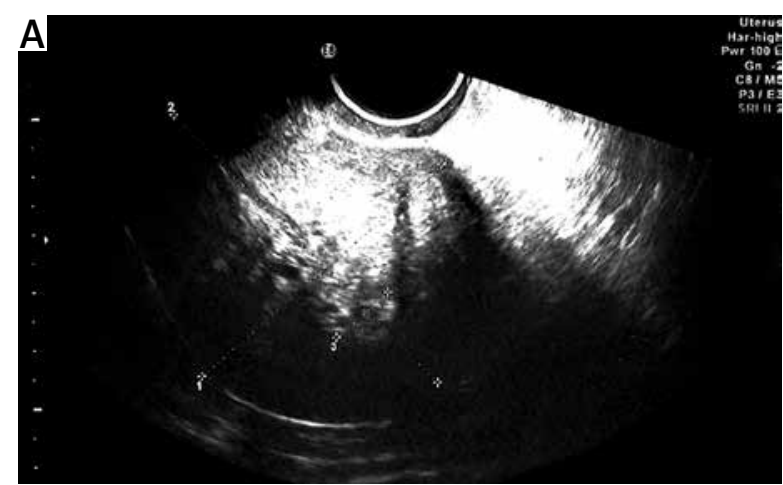

Figure 1. A - Endometrial hyperplasia in TVS. B - Uterine cavity of patient 1 - no signs of hyperplasia uterine bleeding and an oncologist without consultation with our team referred the patient for total abdominal hysterectomy with bilateral salpingo-oophorectomy. As an example we compare TVS scans and hysteroscopy pictures from 2 patients. Patient 1 with suspicion of hyperplasia (pathology - normal endometrium) (Figures $1 \mathrm{~A}, \mathrm{~B})$. Patient 2 with pyometra on TVS (pathology - atrophic endometrium) (Figures $2 \mathrm{~A}, \mathrm{~B}$ ).

All women after hysteroscopic assessment retrieved complete pathology analysis. To date, based on the results obtained after hysteroscopy, 8 of 9 did not have hysterectomy because of suspicion of premalignant stages or malignancy in TVS.

\section{Discussion}

Our preliminary results confirm the value of thorough endometrial assessment in women treated with tamoxifen. Their diagnosis of breast cancer directs them for routine gynecologic consultations, firstly because of suspicion of long-term metastasis in ovaries, secondly when they enter hormone therapy for the evaluation of endometrium.

However, it is a very common finding that patients treated with tamoxifen, if referred to a gynecologist for further evaluation, are referred due to abnormal uterine bleeding (AUB). Most of them in Poland are assigned to undergo blind curettage. This is a result of the small number of hospitals performing hysteroscopic procedures on a 1-day basis. Nevertheless, it should be noted that a study published in 2004 showed that blind biopsies failed to detect 5 of 5 polyps and 33 of 37 cystic atrophies.

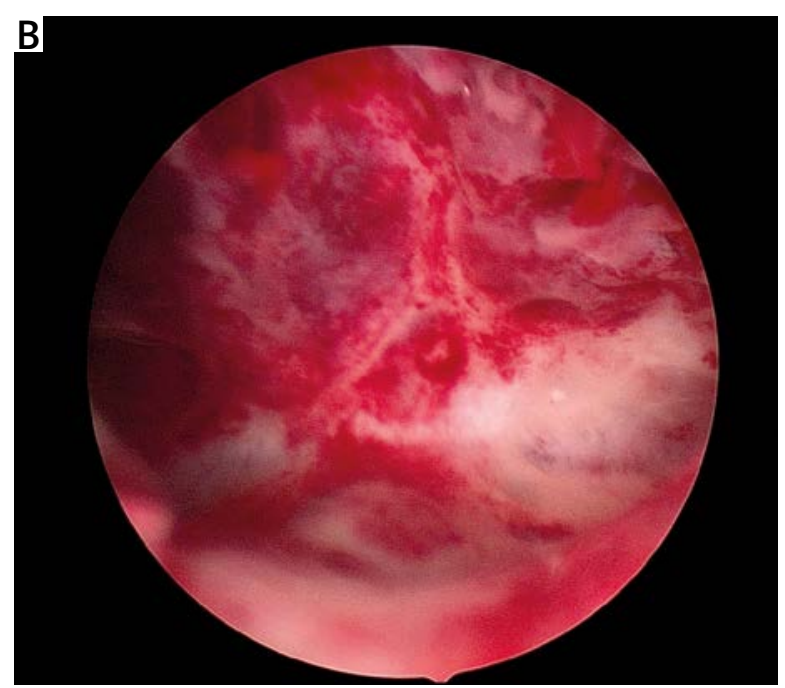




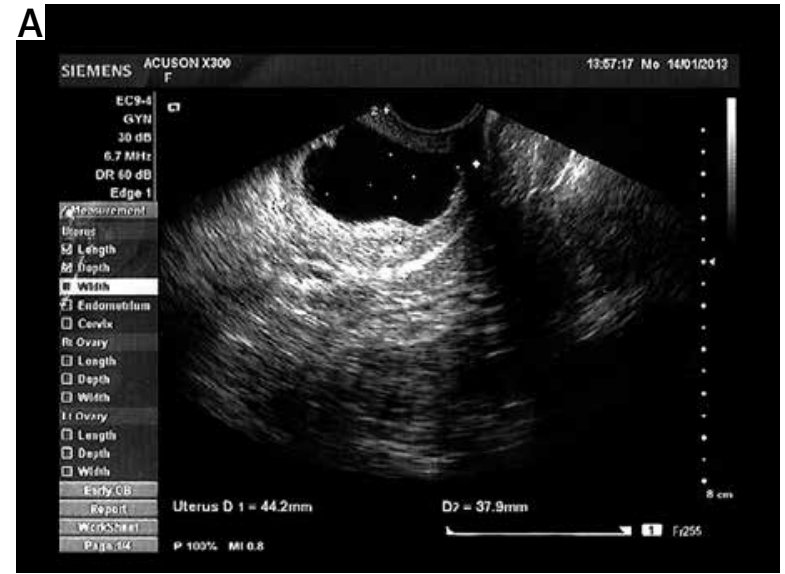

Figure 2. A - Fluid collection in TVS. B - Endometrial lining next to cervical internal os

What is more important, in $34.1 \%$ of cases they provided insufficient tissue for diagnosis; however, no hyperplasia or carcinoma was found. In contrast, all specimens collected under vision were pathologically valuable. Thirty-four of 38 hysteroscopic reports of cystic atrophy were confirmed, and neither endometrial polyps, nor hyperplasia and carcinomas, were undetected. In our preliminary study all cases had definitive pathology diagnosis after directed hysteroscopic sampling. We recruited our group according to groups described in the study of Taponeco et al. [4]. So far, it is impossible in our study to compare the hysteroscopic images for patients taking tamoxifen for less or more than 3 years. We decided to divide our patients into these subgroups as it was previously proven that the incidence of premalignant and malignant lesions in postmenopausal tamoxifen-treated patients longer than 3 years was higher compared to those treated for less than 3 years. Taponeco stated that hysteroscopy should be considered as the first diagnostic procedure to perform in tamoxifen-treated postmenopausal patients presenting uterine bleeding and in postmenopausal women treated for longer than 3 years [4].

More importantly, our preliminary results seem to confirm conclusions suggested in 2002 by the research group in Poznan that there is no exact correlation between ultrasound scan results and pathomorphological findings in patients treated with tamoxifen for breast cancer [5]. However, it is very

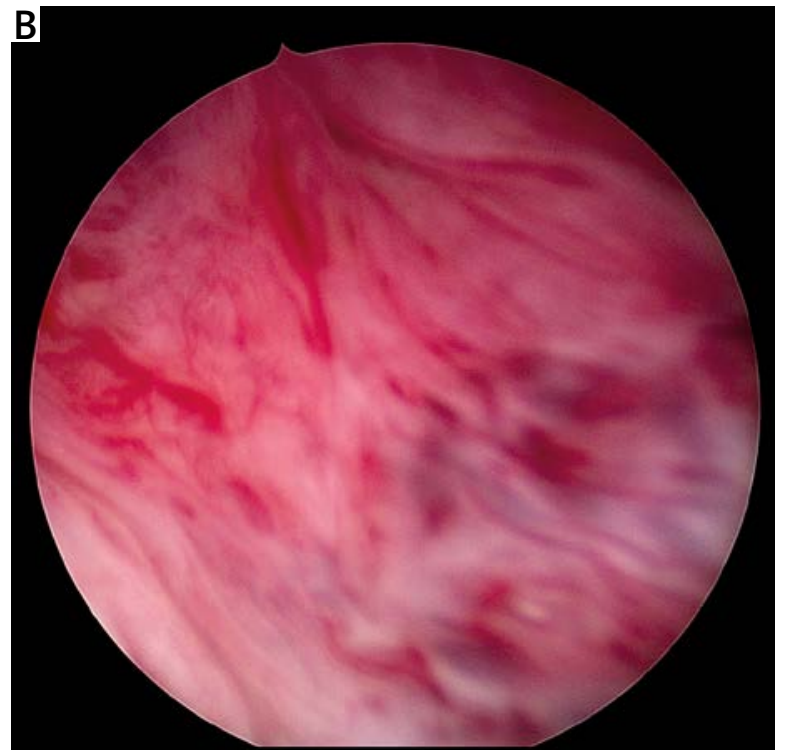

important to enlarge our group with special attention to the patients taking tamoxifen for less than 3 years that we have not recruited so far. It is important to spread among oncologists and gynecologists the value of hysteroscopy, as the main obstacle that we encounter is the lack of information for patients about this possibility and referring patients for routine blind curettage. Therefore, patients are not informed about the significant value of hysteroscopy over TVS or curettage in tamoxifen-treated patients [1-5].

One of the major issues that we encounter in relation with other specialists and patients is the safety of the hysteroscopic procedure. It is obviously skills and equipment-dependent; however, a recent study by Wortman et al. analyzing 305 primary operative hysteroscopic procedures in an office setting showed that only 1 in 305 patients had uterine perforation necessitating diagnostic laparoscopy. This study was performed using a 9-mm operative resectoscope for operative procedures such as endomyometrial resection, myomectomy and polypectomy [6]. On a regular basis, in case of diagnostic assessment of endometrium such as for breast cancer patients, it is usually limited to simple endometrial biopsy.

\section{Conclusions}

Hysteroscopy is a powerful method for diagnosing endometrial disease because it provides a direct view of the uterine cavity, reveals focal lesions and 
enables targeted biopsies to be performed at the same time.

\section{References}

1. Lopez DM, Fernandez YG, Sanchez AV, et al. Baseline hysteroscopicassessement of endometrium in asymptomatic postmenopausal women with estrogen receptor-positive breast cancer. Menopause 2013; 20: 64-71.

2. Gao WL, Zhang LP, Feng LM. Comparative study of transvaginalultrasonographic and diagnostic hysteroscopic findings in postmenopausal breast cancer patients treated with tamoxifen. Chin Med J 2011; 124: 2335-9.

3. Garuti G, Cellani F, Colonnelli M, et al. Hysteroscopically targeted biopsies with blind samplings in endometrial assessment of menopausal women taking tamoxifen for breast cancer. Am Assoc Gynecol Laparosc 2004; 11: 62-7.

4. Taponeco F, Curcio C, Fasciani A, et al. Indication of hysteroscopy in tamoxifen treated breast cancer patients. I Exp Clin Cancer Res 2002; 21: 37-43.

5. Obrebowska A, Sibila-Owczarska A, Sajdak S. Evaluation of endometrium during tamoxifen therapy of breast cancer. Ginekol Pol 2002; 73: 1109-12.

6. Wortman M, Daggett A, Ball C. Operative hysteroscopy in an office-based surgical setting: review of patient safety and satisfaction in 414 cases. J Minim Invasive Gynecol 2013; 20: 56-63.

Received: 16.04.2013, accepted: 10.06.2013. 